\title{
RESET
}

Recherches en sciences sociales sur Internet

\section{Antiracisme ordinaire et (re)catégorisations sociales dans les commentaires d'internautes}

Ordinary anti-racism and social (re)categorizations in Internet users comments

\section{Matthieu Mazzega}

\section{(2) OpenEdition}

\section{Journals}

Édition électronique

URL : http://journals.openedition.org/reset/137

DOI : $10.4000 /$ reset.137

ISSN : 2264-6221

\section{Éditeur}

Association Recherches en sciences sociales sur Internet

\section{Référence électronique}

Matthieu Mazzega, «Antiracisme ordinaire et (re)catégorisations sociales dans les commentaires d'internautes », RESET [En ligne], 1 | 2012, mis en ligne le 30 décembre 2012, consulté le 14 novembre 2019. URL : http://journals.openedition.org/reset/137

Ce document a été généré automatiquement le 14 novembre 2019.

(c) Association Recherches en sciences sociales sur Internet 


\title{
Antiracisme ordinaire et (re)catégorisations sociales dans les commentaires d'internautes
}

\author{
Ordinary anti-racism and social (re)categorizations in Internet users comments
}

\author{
Matthieu Mazzega
}

\section{Introduction}

1 Le phénomène du racisme constitue un champ de recherche historique pour les sciences sociales. La dimension éthique et morale du sujet représente en outre un défi pour les recherches qui visent à en expliquer les fondements ou les conséquences. Dans la sphère ordinaire comme dans la sphère savante, le racisme et son actualité semblent avoir des répercussions sur les manières dont les individus, par ailleurs de plus en plus appelés à s'exprimer sur ce thème, s'approprient et discutent ces questions. Interrogeant l'antiracisme « ordinaire ", cet article étudiera les différentes façons dont l'individu participant à une discussion sur cette question explique et dénonce ce phénomène. Pour cela, nous nous appuierons sur l'analyse d'un large corpus de commentaires d'internautes publiés en réaction à des articles de presse en ligne et touchant, d'une manière ou d'une autre, à la question du racisme. En nous appuyant sur la sociologie phénoménologique d'Alfred Schütz et le courant pragmatique qui s'en inspire, nous analyserons plus spécifiquement les différents processus par lesquels les commentateurs objectivent et donc justifient une explication et une dénonciation du racisme en recourant à des mécanismes de catégorisation sociale, ainsi que les connaissances ordinaires qu'ils mobilisent. Nous commencerons par situer notre démarche au sein des différentes approches du racisme et de l'antiracisme puis nous discuterons les modalités d'ancrage de celle-ci au sein de l'espace particulier des commentaires d'internautes sur les sites des journaux Libération et Le Figaro. Nous nous concentrerons ensuite sur l'analyse proprement dite des types d'argumentations où la référence à la position sociale ou à ses dérivés vient objectiver et légitimer des 
explications particulières du phénomène. À partir de ces différents modes de théorisation et de dénonciation ordinaire du racisme, nous tenterons alors de comprendre le rôle et la spécificité des catégorisations sociales utilisées par les commentateurs.

\section{L'antiracisme comme stock de connaissances ordinaires}

\section{Sociologie du racisme : Définir et circonscrire le phénomène}

2 L'ouvrage Race et Histoire de Claude Levi-Strauss, issu d'un cycle de conférences donné à l'Unesco en 1952, suivi en 1971 de Race et culture, a posé les jalons de la recherche sur le racisme, à commencer par une réponse à la question centrale: «qu'est-ce que le racisme?». Dans ces textes, Lévi-Strauss circonscrit en effet le phénomène par le concept d'«hostilité active », le différenciant alors de l'ethnocentrisme, phénomène qu'il considère comme universel. Pour l'anthropologue, le racisme apparaît dès lors que, au-delà des rapports entre cultures, est prônée ou mise en œuvre la destruction ou l'oppression d'une culture particulière au nom d'une doctrine rapprochant patrimoine génétique et aptitudes intellectuelles, et établissant ainsi une hiérarchie entre les groupes qu'elle constitue. Par symétrie, Lévi-Strauss établit les bases d'une réfutation intellectuelle de ces doctrines en s'appuyant sur un relativisme culturel fort, et en posant l'impossibilité universelle de mesurer, et donc de comparer, les critères propres à une culture particulière. Comme l'a noté, par la suite, Pierre-André Taguieff, l'approche de Lévi-Strauss constitue une «définition forte du phénomène dont la première conséquence est de restreindre l'extension du terme » (Taguieff, 1995 : 10).

Suite à Levi-Strauss, les réflexions d'Albert Memmi proposent une vision différente du phénomène. Effectivement, si le racisme est circonscrit à des phénomènes violents dans le premier cas, l'analyse de Memmi, sur fond colonial, s'attache davantage à le déconstruire à partir des « attitudes » qu'il produit. Le racisme est alors défini comme « la valorisation, généralisée et définitive, de différences, réelles ou imaginaires, au profit de l'accusateur et au détriment de sa victime, afin de justifier ses privilèges ou son agression » (Memmi, 1994 : 14). Dépassant la vision du racisme comme acte violent, Memmi inclut dans l'analyse une dimension cognitive, instituant davantage le racisme comme un système de domination. Pour reprendre la distinction de Mannheim, tandis que Lévi-Strauss tend à renvoyer le racisme à une idéologie, une « doctrine précise », la définition de Memmi touche davantage à ce que Mannheim appelle l'idéologie totale et ses implications en termes d'inconscient collectif (Mannheim, 2006 [1929]). Colette Guillaumin, psychosociologue, étaiera alors ce programme de recherche en déconstruisant «l'idéologie raciste» dans une étude centrée sur l'analyse du langage. Son cadre analytique, organisé autour du couple majoritaire/minoritaire, s'attache à déconstruire l'idée de « race » et ses avatars dans la mise en place et la conservation de ce système inégalitaire (Guillaumin, 1972). Comme pour Memmi, les mécanismes de différenciation et d'essentialisation de cette différence sont pour Guillaumin le cœur de l'idéologie raciste, où ils catégorisent de façon définitive le minoritaire. L'analyse montre alors que le référentiel linguistique est centré sur le majoritaire qui, lui, échappe à toute assignation. Cette étude centrale dans le champ de la recherche sur le racisme a contribué d'une part à mettre en lumière l'enracinement de l'idéologie 
raciste dans les structures cognitives, notamment par le biais d'opérations de catégorisation, et d'autre part à ouvrir la voie à une déconstruction systématique de la fonction de «l'idée de nature » au sein des discours touchant à la différence. En effet, pour reprendre l'auteure, le racisme, dans une définition élargie cette fois-ci, réside globalement dans «l'essentialisation somato-biologique du différent» et dans la « conduite de mise à part revêtue du signe de la permanence» (Guillaumin, op. cit. : 298). Dès lors, le travail de Guillaumin sera poursuivi sur d'autres phénomènes en proie à l'essentialisation et à la hiérarchisation, à commencer par les questions de genre et de sexisme.

4 Les mouvements de redéfinition de la question du racisme dans les sciences sociales françaises peuvent donc s'articuler autour de ces trois figures. Si l'analyse de LéviStrauss a permis de soustraire la question du racisme à une simple "pulsion" en la replaçant dans un cadre intellectuel global, elle a également limité le phénomène à ses manifestations violentes; face à cela Memmi et Guillaumin se sont efforcés de comprendre le phénomène à partir de ses arrière-plans idéologiques et cognitifs. Dans le prolongement de ces travaux fondateurs, la recherche sur le racisme s'est poursuivie dans un certain éclatement: au-delà des problématiques de discrimination, de ségrégation ou même d'intégration, le racisme, dans ses formes contemporaines, tend de plus en plus à s'imbriquer dans des phénomènes connexes, ce qui explique sa fragmentation (Wieviorka, 1998).

\section{Sociologie de l'antiracisme : de l'objet savant à la connaissance ordinaire}

5 Face à cette conceptualisation du racisme, la seconde moitié $d u$ xx ${ }^{\text {ème }}$ siècle voit progressivement naître et s'institutionnaliser un autre mouvement baptisé « antiracisme ». Si les sciences sociales se sont d'abord emparées des discours produits par ses acteurs les plus militants, la prise en compte politique du phénomène du racisme et de ses conséquences, notamment en termes de "discriminations », a ensuite ouvert la voie à l'analyse des implications et des dispositifs politiques se réclamant de ce secteur de l'action publique ${ }^{1}$.

6 Les travaux pionniers de Taguieff portent sur les différents registres qui sous-tendent l'antiracisme militant en France. Son approche engage une reformulation des travaux académiques existants sur le racisme puis une déconstruction systématique des discours de l'antiracisme militant. Plus largement, Taguieff crée et formalise l'antiracisme en tant qu'objet d'étude, à partir d'une approche philosophique de ses fondements. Trois points de son travail semblent capitaux pour notre perspective. D'abord, il identifie et déconstruit un racisme différentialiste ${ }^{2}$, cohabitant avec un racisme « scientifique » qui prend pour pivot l'idée de race. Appuyant sa démonstration sur les analyses de Guillaumin autour des opérations de "naturalisation » propres au racisme, il met en lumière la tendance contemporaine du racisme à naturaliser et à essentialiser l'appartenance culturelle ou ce qui est défini comme tel. Dès lors, il n'est plus nécessairement question de l'infériorité de l'autre mais davantage de sa différence absolue et de son caractère "incompatible » ou « inassimilable ». Conséquence de cette double-identification du racisme, l'exploration du «jeu de miroirs » entre racisme et antiracisme devient nécessaire. Partant du constat d'un dédoublement de l'idéologie raciste et de la perte de vue du racisme «à l'état brut » (Wieviorka, 1998), le travail de 
Taguieff illustre comment les différentes orientations antiracistes en viennent à se superposer avec des types de racisme. Le chercheur en arrive finalement à penser ce jeu d'opposition autour de la dialectique universalisme/particularisme comme la limite infranchissable pour la philosophie antiraciste, au point d'ailleurs, échappant alors à ses constructions symétriques, de préconiser la direction de «l'exigence d'universalité » (Taguieff, $1990: 489)^{3}$.

Dans la perspective qui nous intéresse, les travaux de Taguieff permettent donc de poser les fondements d'une étude de la construction sociale du racisme. En effet, si les auteurs pionniers se demandaient ce qu'était le racisme, Taguieff a poursuivi leurs recherches en ouvrant la question de l'antiracisme et en prenant en compte la place symbolique et morale du racisme dans la société contemporaine. D'une part, en étudiant la diabolisation du racisme, Taguieff prend en compte sa signification en tant que «mal absolu » ou que « survivance archaïque ». D'autre part, il met en lumière la tendance à la disqualification «totale » qu'implique l'accusation de racisme. C'est sur cet ensemble d'éléments, ayant trait au statut symbolique du racisme, que Taguieff pose les bases de l'étude des «idéologies antiracistes », via leur dimension militante.

De notre côté, nous postulons que la prise en compte de la dimension «morale » du racisme rend également possible une approche de l'antiracisme dans sa dimension ordinaire. Ainsi, certaines études ont montré le caractère «tabou » du racisme au sein des discussions ordinaires, et plus spécifiquement les comportements d'évitements que les individus peuvent adopter face à la question du racisme (Eliasoph, 1999; Van Dijk, 1992 ; Bonilla-Silva, 2009). Étienne Balibar a pour sa part mis en avant les conséquences de l'interdit du racisme en termes de contraintes sur le débat, ainsi que la tendance universalisante de la catégorie " racisme " entendu comme sa propension à définir de plus en plus de phénomènes connexes (Balibar, 2005). De façon générale, nous poserons que les discussions faisant intervenir cette question s'avèrent risquées pour les individus y participant par la réprobation morale qu'induit l'accusation de racisme et parallèlement la cohabitation de plusieurs registres explicatifs. Plus encore, le contexte actuel, marqué par une problématisation accrue des questions d'appartenance culturelle et d'immigration, rend de plus en plus fréquentes de telles discussions. Face à cette tension et aux risques qu'elle implique, nous faisons l'hypothèse que l'individu, lorsqu'il fait l'expérience d'une situation intersubjective où intervient la question du racisme, adopte une posture de "pleine attention à la vie et à ses exigences " (wideawakeness ; Schütz, $2008: 110$ ).

Outre l'analyse de la dimension morale et symbolique du racisme et de l'antiracisme, l'approche de Michèle Lamont, ancrée dans la sociologie culturelle, constitue une entrée prometteuse. Celle-ci prend pour objet la rhétorique antiraciste ordinaire pour analyser et comparer certains "répertoires culturels " français et américains utilisés pour catégoriser l'autre, qu'il soit pensé racialement ou socialement. Dans cette perspective, l'antiracisme est défini comme « l'ensemble des répertoires d'arguments et de preuves utilisés pour démontrer que les groupes raciaux sont sur un plan d'égalité " (Lamont, 2000 : 86). Lamont s'appuie alors sur la rhétorique antiraciste des travailleurs de part et d'autre de l'Atlantique pour mettre en lumière formes et contenus des opérations de construction, de maintien ou de suppression des frontières symboliques entre groupes. Cette contribution a largement inspiré notre étude, notamment parce qu'elle ancre l'antiracisme dans un contexte national à partir d'une sociologie des opérations de "meaning-making " - à comprendre comme l'étude de la construction 
sociale du savoir - déployées par les individus ordinaires. Nous nous appuierons en particulier sur le concept transversal de frontières symboliques entendu comme « les types de démarcation que les individus tracent quand ils catégorisent les gens" (Lamont, $1995: 24$ ).

10 À la croisée de ces approches, notre étude de l'antiracisme ordinaire a pour ambition de comprendre comment les individus «parlent » le racisme en utilisant des arguments et des connaissances jugés légitimes et échappant a priori à de possibles accusations de racisme. Nous inscrivons alors notre cadre analytique dans une sociologie de la connaissance ordinaire qui vise à identifier le "stock de connaissances " (Berger \& Luckmann, 1986) et les critères de pertinence (Schütz, 2008) utilisés par les individus lorsqu'ils font l'expérience d'une situation de ce type. L'analyse cherche à identifier les interprétations prises comme allant de soi, et à comprendre comment certaines constructions argumentatives peuvent entrer en conflit: en d'autres termes, les schèmes institutionnalisés d'un côté et ceux qui nécessitent une légitimation de l'autre. C'est là par ailleurs que notre approche diffère de celle de Lamont, dans la mesure où nous souhaitons appréhender le recours aux connaissances disponibles " en situation ", c'est-à-dire dans une dimension interindividuelle. Il s'agit donc de modéliser les argumentations des individus se trouvant dans une situation particulière - où la question du racisme est abordée de façon intersubjective - et de comprendre les différentes typifications et structures de pertinence employées pour interpréter et catégoriser la situation et ses divers éléments. Le stock de connaissances ainsi reconstitué fonde alors, de façon idéal-typique, ce que nous nommons globalement l'antiracisme ordinaire.

\section{De l'antiracisme ordinaire dans les commentaires d'internautes}

11 La dimension empirique d'une telle étude n'est pas sans poser problème au vu des contraintes et des risques individuels mentionnés plus haut, et ce d'autant plus que nous cherchons à étudier l'objet dans sa dimension interindividuelle. Face à cette difficulté, nous avons choisi d'approcher notre objet au sein d'un espace particulier, celui des commentaires d'internautes sur deux sites de presse, liberation.fr et lefigaro.fr, créés respectivement en 1997 et 1996. Par leur inscription progressive dans ce qu'il est coutume d'appeler le web 2.0, le site Libération a mis en place un système de commentaires d'internautes durant l'été 2006 et fut suivi de peu par Le Figaro. Ce dispositif a fait l'objet d'études portant sur le lien entre producteurs et consommateurs d'informations, et dans une certaine mesure, sur la «coproduction» de l'information ${ }^{4}$. Dans cet article, nous laisserons de côté ces problématiques pour nous concentrer spécifiquement sur les conditions de construction et d'expression de notre objet d'étude, ce qui nécessite tout d'abord de justifier le corpus retenu et l'opportunité qu'il représente pour une analyse de l'antiracisme ordinaire.

Plusieurs raisons expliquent le choix de ces deux journaux. D'une part, ils connaissent les plus fortes fréquentations parmi les sites où la participation - et donc le commentaire - n'est pas payante ${ }^{5}$. D'autre part, malgré l'absence d'études sur la traduction dans l'espace numérique des clivages médiatiques traditionnels, ils permettent de prendre en compte l'effet éventuel du clivage politique gauche/droite, même si nous notons qu'a posteriori, nos résultats ne font pas apparaître de différences majeures dans l'argumentation des internautes entre les deux plateformes. En ce qui 
concerne le choix des articles et des commentaires étudiés, nous avons évoqué plus haut les réflexions de Taguieff relatives à la multiplication des formes de racisme et plus brièvement celles de Wieviorka mettant en avant les tendances à l'imbrication contemporaine du racisme avec d'autres thématiques (Wieviorka, 1998). Nous avons donc inclus dans notre analyse un grand nombre d'articles relevant de phénomènes qui, d'une manière ou d'une autre, convoquent la question du racisme, parce qu'ils abordent, individuellement ou collectivement, la question de l'autre et de sa différence, réelle ou imaginée. Les thèmes des articles choisis vont ainsi de violences ou de propos jugés racistes jusqu'aux déclarations politiques sur l'idée de multiculturalisme, en passant par la mise en forme politique des questions identitaires et culturelles, à travers l'extrême droite ou les divers débats nationaux sur l'immigration et l'Islam. Ces critères de sélection ont permis de constituer un corpus d'environ 12000 commentaires en réaction à 22 articles extraits des deux sites étudiés (cf. Annexe). Cette définition large des commentaires pris en compte dans notre étude vise à identifier les connaissances et constructions ordinaires qui traversent l'ensemble des cas retenus. Plus particulièrement, pour rependre la phénoménologie de Schütz, en reconstruisant les critères utilisés par les internautes pour interpréter indistinctement ces situations, nous pourrons poser que celles-ci sont objectivées, et donc typifiées, sous un même « horizon de sens» (Schütz, 2007, 2008). L'antiracisme ordinaire que nous cherchons à déconstruire ici correspond alors au stock de connaissances ordinaires en d'autres termes le schème de référence - disponible pour définir ce type général d'expérience, à savoir " parler le racisme » sur le terrain particulier des commentaires sur Internet.

13 Dans les commentaires d'internautes, l'antiracisme prend d'abord une forme langagière. La forme écrite du commentaire renvoie à « un système de signe de second degré » (Berger \& Luckmann, 1986 : 93) prenant son origine dans des relations de faceà-face. La dimension interactive des commentaires doit également être abordée, ce que permet le cadre interactionniste (au sens d'Erving Goffman) d'analyse des interactions particulières. Ainsi, en prenant en compte l'ensemble des commentaires touchant à un article, nous avons affaire à une production écrite collective. En premier lieu, le dispositif du commentaire consiste à donner un avis sur l'événement relaté par l'article; plus spécifiquement, les commentaires portent davantage un avis sur la question soulevée par l'évènement que sur l'article en tant que tel. Ils peuvent aussi consister à compléter l'article - et le sujet traité - par un témoignage biographique ou encore par la mention de sources supplémentaires. Parallèlement à ce premier type de relation article/commentateur, la production d'un commentaire peut également viser à donner un avis sur un autre commentaire ou groupe de commentaires. Nous poserons généralement que le commentaire est produit par et pour une relation entre son auteur, le contenu de l'article commenté et les autres commentaires présents. L'expérience du commentateur consiste alors à rédiger et ainsi à exprimer sa propre identification et interprétation de la question soulevée par l'article commenté, en se basant explicitement sur ce dernier ou en contestant ou appuyant la position d'un ou plusieurs commentaires préexistants. En retour, le commentaire étant publié et appartenant de ce fait à la discussion collective, il peut lui-même devenir le point d'appui d'un autre commentateur et ainsi de suite. Cette production cumulative se présente sous la forme d'un fil principal où cohabitent commentaires mêlant interprétations générales de différentes questions posées par l'article et métainterprétations - c'est-à-dire interprétations des interprétations d'autrui - et, de part 
et d'autre de ce fil, le développement de fils secondaires au sein desquels un petit nombre de commentateurs se répondent entre eux ${ }^{6}$.

Par ailleurs, cet espace et les échanges qu'il produit restent avant tout intersubjectifs et relèvent en cela d'un monde de culture. Pour le dire différemment, les objets - c'est-àdire les constructions argumentatives qui font sens pour les acteurs - qui balisent la discussion produite par l'ensemble des commentaires, appartiennent d'une manière ou d'une autre à un cadre culturel particulier, ici le contexte français ${ }^{7}$. En cela, on peut postuler que l'individu participant à cette expérience, notamment lorsque celle-ci s'apparente de façon typique à "parler le racisme", possède un stock de savoirs disponibles à la fois pour interpréter la question soulevée par l'article mais également pour comprendre et juger les contributions d'autrui. Toutefois, contrairement à la réalité quotidienne, entendue comme un flux ininterrompu et spontané, la temporalité discontinue des commentaires et l'absence de face à face réel autorisent selon nous un horizon de choix plus importants - et plus risqués - pour l'individu et un usage particulier du stock de connaissances disponibles. Pour reprendre les termes de Luc Boltanski, nous qualifierons les commentaires étudiés de constructions relevant d'un registre méta-pragmatique, c'est-à-dire où « la relation entre les formes symboliques et les états de choses et, par conséquent, l'espace qui les sépare ou peut les séparer, leurs écarts possibles, leur distance éventuelle, vient se placer au centre des préoccupations communes»(Boltanski, 2009: 111). Nous nous intéresserons principalement aux commentaires qui justifient leurs arguments par des opérations de montée en généralité ou de montée en singularité (Boltanski \& Thévenot, 1991) c'est-à-dire qui effectuent, de manière explicite ou non, des aller-retour entre une situation particulière et des formes symboliques plus générales. Notons que les argumentations peuvent procéder dans un sens comme dans l'autre, considérant l'événement relaté par l'article comme appartenant à un type particulier ou partant d'emblée de ce type particulier en y attachant une situation particulière, biographique par exemple. Dans tous les cas, les commentaires analysés font apparaître les justifications et registres dans lesquels ils ancrent leurs propos. En des termes phénoménologiques, ils objectivent la situation. Nous postulons qu'ils dépassent alors la simple réaction à un article précis pour devenir des constructions argumentatives typiques. Pour revenir sur la question du racisme, présente de façon intersubjective dans les événements relatés par les articles retenus, l'analyse se concentrera sur les façons dont les individus, via leurs commentaires, utilisent, justifient ou contestent tel ou tel schème de référence faisant intervenir l'idée de position sociale dans l'interprétation et la qualification du racisme.

Pour terminer, il nous faut définir l'espace des commentaires sous l'angle de ses normes, celui-ci n'échappant pas à certaines modalités de contrôle, particulièrement lorsqu'il est question du racisme. Tout d'abord, d'un point de vue formel, les interfaces de commentaires sont soumises à un système de modération. Réunies dans la charte des commentaires accessible à tous, les conditions de modération mentionnent un ensemble de règles que les participants doivent respecter s'ils veulent voir leur commentaire accéder à l'espace public. La charte interdit tout propos comportant des « incitations à la discrimination, à la haine ou à la violence » (Le Figaro) ou bien encore des « commentaires diffamatoires, racistes, pornographiques, pédophiles, incitant à délits, crimes ou suicides " (Libération). D'un point de vue pratique, la sélection des propos à modérer se fait principalement à partir d'une définition juridique - et donc assez large - du racisme ${ }^{8}$, choix renvoyant en partie au fait qu'en cas de publication d'un commentaire jugé «raciste» aux yeux de la loi, le site peut être poursuivi. 
L'activité s'effectue alors à partir d'une identification automatique de mots-clés mais également via des modérateurs humains jugeant de la conformité des propos à partir d'une charte établie entre le site et la société de modération'. Ainsi, dans le cadre de discussions touchant à la question du racisme, si l'activité de modération, effectuée de manière automatique et/ou humaine, ne laisse pas apparaitre les propos "racistes " juridiquement parlant, il n'en reste pas moins que les participants peuvent viser et sanctionner des messages qui, selon eux, relèvent du racisme. Plus encore, si l'on revient sur les difficultés à définir le phénomène, tant au niveau scientifique qu'au niveau juridique d'ailleurs, les propos passant le filtre de la modération peuvent bien évidemment relever, de près ou de loin, d'idéologies racistes particulières. Poursuivant notre analyse en termes de connaissances ordinaires, notre travail consistera justement à modéliser les raisons et les façons dont les commentateurs étiquettent, dénoncent ou défendent des positions comme « racistes».

Pour conclure, sur cet espace entièrement textuel et dont on ne sait rien a priori de ses interlocuteurs, seul le langage symbolique - au moyen de l'écriture - et ses constructions abstraites et détachées de l'ici et du maintenant (Berger \& Luckmann, 1986 : 89) sont disponibles pour qualifier le racisme d'une part et les participants présents de l'autre. C'est en cela que le corpus retenu correspond initialement à un ensemble de constructions de sens commun, reliant singulier et général, à partir duquel nous chercherons à reconstruire le stock de connaissances disponible lorsqu'un individu est amené simultanément à qualifier la question du racisme et à interpréter les positions d'autrui sur cette même question.

\section{Théorisations et dénonciations ordinaires du racisme par la position sociale et ses dérivés}

17 Notre analyse du corpus conduit à identifier trois registres sur lesquels se construisent une grande majorité des commentaires retenus : la classe, la nation et la race. Nous nous proposons ici d'explorer le premier d'entre eux au travers des façons dont la référence ordinaire à l'idée de classe apparaît dans le corpus. Le travail d'identification a débuté par une analyse complète d'un sous-corpus de trois articles et de leurs commentaires. À partir de là, nous avons retenu une liste de constructions désignant, directement ou non, la position ou la structure sociale. Ces constructions renvoient à des qualifications socio-économiques (riche, pauvre, bourgeois, populaire, métier...) ou à des qualifications reposant sur des formes plus symboliques (dominés/dominants, élites, masses...). À l'aide d'une identification lexicométrique effectuée sur l'ensemble des articles retenus, nous avons reconstruit un corpus à partir de la totalité des commentaires comportant ce type de constructions. Celui-ci ne comportant plus que des commentaires détachés de leur article initial, il devient davantage figé autour de constructions typiques reliant catégorisations sociales et racisme. Ainsi, s'il perd en contextualité et en interactivité, il devient un ensemble homogène rassemblant une série de constructions anonymes et objectives. C'est la raison pour laquelle nous ne mentionnerons pas les références (auteur/article/journal) des commentaires utilisés pour illustrer le propos. Après avoir codé l'ensemble du corpus, nous avons retenu uniquement les commentaires présentant des montées en généralité ou en singularité, c'est-à-dire mettant en place des ponts entre singulier et général. Après réduction des catégories, nous avons alors pu identifier deux types d'usages particuliers de la classe 
sociale, ou du moins des catégories sociales : d'une part l'explication du racisme par la structure sociale et les implications morales de cette dernière, et d'autre part la dénonciation du racisme par deux « figures » sociales.

\section{De la distribution sociale du racisme : racisme populaire et racisme des élites}

Le premier type de construction renvoie aux commentaires qui expliquent le racisme à partir de la réappropriation de connaissances liées à la stratification sociale. Notons d'emblée que la représentation de cette dernière est largement polarisée par les commentateurs autour de la "classe populaire» et des «élites». Dès lors, les théorisations $\mathrm{du}$ phénomène $\mathrm{du}$ racisme passent principalement par des catégorisations touchant à l'une ou l'autre de ces constructions. Nous présenterons successivement ces deux types de théorisation du racisme en les illustrant par des commentaires typiques ${ }^{10}$.

Le premier registre analysé concerne les commentaires dont l'argumentation, de manière plus ou moins construite, évoque les relations entre racisme et classe populaire. Leur problématique consiste à expliquer pourquoi les individus supposés appartenir à cette classe manifestent davantage de réactions ou de pensées considérées comme " racistes » : cette question initiale est construite à partir d'un constat allant apparemment de soi - la propension plus importante de la classe populaire à manifester du racisme - et les messages se rapportant à cette orientation cherchent à justifier une telle position en usant de connaissances plus larges et tenues pour objectives et cohérentes. En d'autres termes, les commentaires s'attachent à "théoriser " le lien entre racisme et classe populaire et ce, notamment, en usant de constructions symboliques et morales concernant la stratification sociale et son fonctionnement. La majorité des commentaires appuie ainsi son interprétation du lien racisme/classe populaire par des références à «la peur économique » inhérente à la réalité contemporaine. L'évocation de la crise financière et économique de 2009 est alors une référence typique dans les contributions en question comme dans le commentaire suivant :

La vérité qui me parait la plus plausible, c'est que la crise est un facteur d'affaiblissement des esprits ; face à ça, la remise en cause du Système économique étant impossible, on se met à reprocher à une communauté son inhumanité, et on sent venir la même catastrophe qu'en 1939.

Dans ce propos, l'argumentation qui sous-tend le message autour de la crise et de «l'affaiblissement des esprits" renvoie symboliquement à la classe populaire qui, logiquement, s'avérerait la plus touchée par la crise. Le mécanisme de «montée en généralité » (Boltanski \& Thévenot, 1991) passant par la remise en cause globale du "système économique » est caractéristique des commentaires de ce registre. Dans un impératif de justification, opéré par un processus d'objectivation du lien entre racisme et classe populaire, cette montée en généralité semble être utilisée pour se démarquer d'une explication qui fait du lien racisme/classe populaire un facteur «naturel ", à l'image du commentaire suivant.

Si l'on ne prend pas cela en compte [la crise], on va chercher à expliquer une situation de désarroi où le populo est guidé par des pulsions irrationnelles puisqu'il n'est pas en situation de prendre des décisions calmement puisqu'il a peur en l'avenir...; Il y aura toujours des gens qui auront peur de l'autre, mais la 
généralisation de ce sentiment est principalement due à la situation précaire dans laquelle la société actuelle est. Les musulmans ne sont guère plus qu'une soupape de sécurité pour évacuer cette peur. S'ils n'étaient pas là, on aurait trouvé autre chose...

21 Les commentaires appartenant à cette catégorie expliquent la relation de causalité entre phénomène du racisme et classe populaire sur le mode du «parce-que » (Schütz, 2007 : 74), c'est-à-dire à partir de la situation et de l'expérience biographique projetées sur les acteurs appartenant à cette classe. Cependant, la justification de ce lien ne peut se satisfaire de la simple référence à la situation économique et sociale. Il s'agit également de savoir pourquoi les membres supposés de la classe populaire seraient plus à même, individuellement, de développer des comportements ou des opinions racistes. Dès lors, les théories ordinaires relevées rappellent la théorie de la personnalité autoritaire: "l'ignorance des complexités de la société contemporaine provoque un état d'incertitude et d'anxiété générales, qui constituent le terrain idéal pour le type moderne de mouvement de masse réactionnaire » (Adorno, 2007 : 231). Elles peuvent se prolonger dans des réflexions en termes de valeurs ou de capital culturel :

Le problème n'est pas le racisme, il n'est finalement que la conséquence du repli lié à la crise économique et le bourrage de crâne des médias. [...] C'est l'individualisme et le matérialisme de nos sociétés, couplés avec les abus caractérisés des élites qui font perdre toute confiance en l'avenir et favorisent le rejet de l'autre sous toutes ses formes.

Comment peuvent s'exprimer dans un tel contexte les pulsions xénophobes, qui, pour être minoritaires, n'en sont pas moins une composante universelle du ressentiment traditionnel des gens politiquement incultes et mécontents du sort qui leur est fait, particulièrement en temps de crise économique? Simplement en instrumentalisant les valeurs républicaines d'égalité et de laïcité [...] Il ne s'agit pas d'une insulte, mais du simple fait de rester prisonnier des pulsions archaïques, telles que la xénophobie, sans avoir conscience des causes de ces affects de ressentiment.

La manière dont les montées en généralités décrites plus haut, bâties sur une critique "globale» du système, renvoie ici, indirectement ou non, à des mécanismes de catégorisation en termes de moralité et de culture. Ce n'est donc plus nécessairement la simple position socio-économique qui explique la propension au racisme mais également les valeurs morales. Établissant un lien entre classe populaire et réactions racistes à partir d'une théorisation construite comme neutre et objective - la conjoncture économique et ses frontières socio-économiques-, les commentaires renforcent par ailleurs ce lien en catégorisant «les gens » appartenant à cette classe supposée dans des termes moraux et symboliques. Que ce soit à partir de l'« inculture », du matérialisme ou encore de l'absence de recul - vis-à-vis des médias par exemple -, les commentaires analysés laissent voir un travail important de marquage des frontières. Ainsi, de la dénonciation du système en général, et économique en particulier, et de ses conséquences sur certains individus « mécontents de leur sort » en termes de rejet de l'étranger, les critiques développées renvoient in fine à toute une série de critères moraux - et subjectifs - pour désigner et catégoriser les personnes appartenant à cette classe, et par analogie les désigner comme responsables typiques du racisme.

Si le premier registre prenait pour point de départ implicite l'idée d'un racisme apparemment inhérent à la classe populaire, le second va davantage axer son propos sur le pragmatisme et le réalisme de celle-ci à partir d'une explication basée sur sa 
relative proximité avec les populations étrangères. La délinquance des minorités devient le centre des contributions avec la mise en avant d'une certaine expérience visà-vis de celle-ci.

Quand vous vivez en banlieue, ce ne doit pas être votre cas, vous trouvez à $23 \mathrm{~h}$ des enfants de 7, 8 ans qui jouent au ballon dans votre hall d'entrée, des jeunes qui à $2 \mathrm{~h}$ $\mathrm{du}$ matin, assis sur le capot de votre voiture écoutent de la musique avec un volume maximum, vous pouvez comprendre que les habitants en ont marres, et qu'ils fuient ces quartiers a la première opportunité, laissant ces populations entre elles. A part ça, effectivement mon médecin est d'origine vietnamienne, mon cardiologue et mon dentiste sont d'origine algérienne. L'un n'empêche pas l'autre

Le message marque d'emblée l'accentuation par l'auteur de sa proximité avec les comportements qu'il entend décrire. Notons au passage que la référence aux minorités est implicite et que le message, implicitement là aussi, individualise la relation en faisant porter sur des individus singuliers, et non sur le groupe, le rejet.

L'argumentation soutenue vise donc à réfuter le lien entre racisme et classe populaire tel qu'il est théorisé par le premier groupe. Par le biais d'une «montée en singularité » cette fois-ci (Boltanski \& Thévenot, 1991), le propos insiste sur l'expérience de son auteur afin de dénoncer certains comportements sans pour autant adhérer à des pensées racistes, preuve en est de ses relations avec des personnes étrangères, "l'un n'empêchant pas l'autre ». C'est alors le réalisme des personnes issues de la classe populaire qui tend à être mis en avant et opposé à un certain « aveuglement » des élites ou autres classes supérieures qui, lui, est dénoncé.

On aimerait que nos élites françaises qui habitent Paris voient la France au delà du périphérique. Une journaliste avait lâché : "Nous avons peur d'aller en banlieue". Et ce sont les mêmes qui nous donnent des leçons sur la vie en banlieue et les bienfaits du multiculturalisme....Si ces élites venaient faire un tour à Aulnay, Villiers le Bel, Bondy, elles verraient la réalité du multiculturalisme, encouragé par les élus locaux, entre nous soit dit. Ces magasins où l'on ne parle plus le français, décorés aux couleurs du pays "là-bas".

Plus particulièrement dans ce dernier commentaire, l'aveuglement des élites, illustré par leur éloignement géographique des lieux où se côtoient classe populaire et populations immigrées, s'accompagne d'une dénonciation de leur côté «donneur de leçon ». Contrairement au premier registre étudié, le second a tendance à théoriser le lien entre racisme et classe populaire à partir, d'une part, de l'expérience de ses membres, qui assisteraient à des comportements inacceptables de la part d'individus appartenant aux minorités, et d'autre part en réfutant le lien entre le fait de dénoncer cela et l'adhésion à des croyances racistes. À partir de là, comme pour le premier axe, on assiste au passage d'une théorisation particulière - bien que la définition des comportements dénoncés soit, elle, subjective - à la création de frontières entre classe populaire et élites. Assortis de références à des critères moraux, notamment réalisme versus aveuglement, qui viennent situer ces frontières, les commentaires vont jusqu'à en appeler à la question de l'honnêteté.

Je suis toujours surpris de cette analyse reprise en boucle par ces penseurs géniaux qui n'ont pas d'autres explications que l'aversion naturelle inée du français, qui est foncièrement méchant, intolérant, haineux,raciste, [...] juger impardonnable les agressions des uns et fermer les yeux sur les agressions des autres, intimer l'ordre au français de souche de se la fermer en le taxant de raciste dès qu'il l'ouvrait . [...] mais continuez donc à considérer les français comme vous le faite à avoir une vision unilatérale des choses, avec la lâcheté qui vous caractérise de ne pas être en mesure de faire la part des choses en généralisant. 


\section{de Richard Hoggart (1991), est utilisée dans le propos, plus encore, c'est l'honnêteté des} élites ou en tout cas leur capacité à « faire la part des choses» qui est mise en doute. Contrairement au premier registre, le racisme des élites est alors défini dans un motif « en vue de » (Schütz, 2007 : 74), c'est-à-dire utilisé pour disqualifier la classe populaire. Le propos lui oppose implicitement l'honnêteté et le franc-parler de cette dernière, dénonçant la lâcheté des classes dominantes. Nous voyons ainsi que les commentaires dont il est question renvoient également à tout un ensemble de critères moraux qui semblent fixer la séparation entre classe populaire et élites. Ces mêmes critères sont justifiés par des explications particulières du lien - minimisé - entre classe populaire et racisme, fondées sur son expérience particulière du phénomène.

Cette première partie de l'analyse laisse voir l'une des façons dont les références à la position sociale s'imbriquent dans des théorisations du racisme. En un sens, les commentaires décrits ici se disputent la distribution sociale du racisme. Nous entendons par là les manières dont la position sociale déterminerait l'usage de schèmes participant au racisme. Aussi, les deux registres utilisés se répondent l'un l'autre. Si le premier part d'un lien présupposé entre racisme et classe populaire, le second fonde son argumentation sur la réfutation - ou la relativisation- de ce présupposé. Particulièrement visible dans le second registre, cette intégration de l'appartenance sociale des individus « racistes » dans l'explication du racisme s'avère également liée à la négociation de ce qu'est - et de ce que n'est pas - le racisme. L'opposition se retrouve alors dans les critères de pertinence utilisés pour construire l'explication: rationalisme/empirisme, motif parce-que/motif en-vue-de, montée en généralité/ montée en singularité. Cependant, dans les deux cas, on assiste à une objectivation du phénomène du racisme à partir du rapport du commentateur à une position sociale particulière puis à son ancrage dans un système de frontières morales séparant classe populaire et élites.

\section{Dénoncer le racisme par des «figures » sociales : les cas du beauf et du bobo}

Si les théorisations ordinaires relevées plus haut conduisent les individus à construire et reconstruire des frontières sociales entre groupes, notamment par la mise en avant des critères moraux jugés légitimes ou non, nous pouvons également relever des constructions qui utilisent directement ces frontières symboliques à travers deux catégorisations - ou plutôt assignations - apparaissant dans les commentaires : celles $\mathrm{du}$ «beauf» et du «bobo». Ces deux expressions sont présentes de manière transversale dans le corpus. Comme pour les catégories précédemment citées, nous abordons celles-ci en tant que catégorisations ordinaires et exogènes, et non en tant que catégories d'analyse. Il s'agit de suivre les manières dont les participants construisent ces catégories et en aucun cas d'investir nous-même ces objets. Or, ces termes apparaissent presque exclusivement dans les commentaires critiques soit envers l'événement relaté par l'article, soit envers d'autres commentaires. Ils incarnent ainsi davantage des figures accusatrices et disqualifiantes pour le destinataire: ces figures sociales renvoient avant tout à des mécanismes de dénonciation morale. Il en va ainsi des deux commentaires suivants, usant respectivement de la figure du bobo et de celle du beauf. 
Pourquoi "dérape" ? Ce Monsieur a tout simplement exprimé, de façon exagérée et caricaturale, bien sûr, ce que la majorité du peuple pense !!!! Il n'y a que la classe politique bien pensante et les bobos caviar, à l'abri du besoin dans leurs cabinets feutrés, qui ne soient pas capable de voir et de dire la vérité... C'est tellement plus facile de faire de l'angélisme et de se bercer d'illusions !!

Violents peut être, mais comment dire,...vos propos lénifiants et vos lieux communs, on en a tellement assez...vos brèves de comptoir...vos principes de grosse beauf...vous me faites gerber madame. L'incitation à la haine vient au départ de vous et de tous les veaux qui votent pour n'importe qui.

Ces deux contributions, qui visent respectivement un article relatant un "dérapage raciste » et un autre commentaire permettent de reconstituer les contenus moraux des deux figures convoquées. Celle du «bobo» se caractérise par une condition socioéconomique aisée. Les références au « caviar » et aux « cabinets feutrés » marquent une catégorisation euphémisée du capital économique. Contrairement à la seconde, celle-ci comporte une dimension géographique forte, les centres villes ou quartier huppés. De l'autre côté, le «beauf» est construite initialement autour de l'idée d'inculture, le «manque de recul » revenant aussi très souvent dans les usages de ce terme. À l'inverse du bobo, la figure du beauf est déterritorialisée, comme une des rares contributions sur le sujet l'a avancé (Guenif-Soulamas, 2003: 65) et n'est pas rapportée explicitement à une condition économique particulière. On voit d'ores et déjà la proximité de ces figures avec les contenus des frontières décrites précédemment. Toutefois, dans ces commentaires, les théorisations du racisme ne sont pas présentes en tant que telles: c'est davantage l'emploi de ces catégories et des frontières socio-économiques, morales et culturelles qu'elles sous-tendent qui laisse entendre une certaine explication du racisme. Des critères moraux surgissent explicitement dans certains propos et correspondent à ceux observés plus haut. Pour ce qui est du bobo, les traits dénoncés renvoient notamment au manque d'honnêteté et à la distance ou ignorance des « vrais » problèmes et donc des solutions à y apporter :

$C^{\prime}$ est la VERITE qui ne plaît pas aux faux culs de la gauche "bobo's", vous savez cette fausse gauche bourgeoise, bienveillante avec les racailles et à des années lumière des préoccupations du monde ouvrier!

Parce que ce n'est peut-être pas un problème, mais vous habitez sur la lune ? Avezvous seulement vu un camp de Rom, en avez-vous seulement rencontrés? Visiblement non! du blablabla de bobo bien à l'abris dans son bunker de bons sentiments

Du côté de la figure du beauf, les traits moraux décriés consistent davantage dans l'idée de fermeture et d'absence d'esprit critique :

Je n'ai pas parlé de français moyen, j'ai parlé de: - beauf français, moyen... Le français moyen a tout mon respect. Le beauf français, moyen, celui qui est incapable d'ouvrir un opuscule pour connaître le meilleur moyen (français ou pas) de frapper sa femme, je n'ai que mépris pour lui...

Quand je vois la qualité des interventions, sur ce même forum de Libé, des militants OUEMPETEUX et autre raclures Frontistes, oui, la parole raciste, l'expression naturelle du beauf de droite....SE DECHAINE sous sarko. Normal quand l'exemple est donné d'en haut. Mais « azerty » à ses oeillères et tout va bien dans le meilleur des monde... Pathétique petit soldat de la sarkosie

On observe la proximité entre les frontières symboliques utilisées par les participants pour situer ces deux figures et celles décrites dans la partie précédente. Par ailleurs, la dénonciation à partir de critères socio-économiques, culturels ou moraux s'inscrit plus généralement dans les marquages de frontières particulières entre l'auteur et la figure qu'il dénonce. On peut ainsi énumérer les catégories principalement utilisées: 
réalisme/idéalisme, honnêteté/malhonnêteté, fermeture/ouverture, critique/noncritique, culture/inculture. Pour revenir aux remarques faites auparavant, on retombe finalement, par le biais de ces deux figures, sur les mêmes critères délimitant le partage entre classe populaire et élites. Cependant, le recours de la critique à ces deux figures nous semble avoir des conséquences sur la dénonciation sociale du racisme. En effet, si les théorisations décrites prenaient pour appui des arguments ou des termes renvoyant à des thèmes et configurations plus classiques et stabilisés - l'idée générale d'une structure sociale - l'usage et le contenu symbolique de ces deux figures échappent dans une certaine mesure à une hiérarchisation a priori. Dès lors, certaines opérations visent à contester et à renverser des répertoires et catégorisations sociales classiques. Le couple réactionnaire/progressiste se trouve par exemple disputé entre ces deux figures :

C'est comme tous les bobos de ce site qui répètent à l'envie, histoire de donner mauvaise conscience à certains, que les éboueurs sont noirs... obsédés par la couleur de peau ils sont (avec en plus un retard de quelques années sur la réalités des choses)

Les gros blaireaux racistes réac xénophobes comme toi trouvent le moyen d'ouvrir leurs bouches, pour nous expliquer que mais non c'est un brave homme, comprenez le ! [...] des gros beauf comme toi [...] tu n'es pas ma France toi tu es un résidu qui à terme si le pays tournait mieux devrait disparaitre comme les dinosaures

À partir des propos reproduits ici, on peut donc voir partiellement le rôle de ces deux figures dans la capacité des personnes à remettre en cause certaines catégorisations. C'est le cas avec la position « réactionnaire » qui est successivement attachée à la figure du bobo, «non-réaliste » par ailleurs, et à celle du beauf, associée à sa « bêtise ».

Si cette rapide modélisation des constituants des figures du bobo et du beauf montrent que celles-ci font se rejouer de façon métaphorique et/ou euphémisée l'opposition élites/classe populaire, le travail ordinaire de catégorisation et de marquage de frontières nous semble toutefois plus « ouvert » : ces figures offrent des possibilités de critique et de dénonciation bien plus importantes. Ainsi, à partir de ces figures et de leurs ancrages moraux et symboliques, la simple assignation de l'une ou de l'autre semble suffire pour dénoncer la responsabilité de son interlocuteur dans le phénomène du racisme et ce sans devoir adosser explicitement - et donc justifier - sa critique à une théorisation du phénomène.

\section{Racisme, moralité et positions sociales}

L'étude des processus par lesquels l'idée de classe sociale est mobilisée dans les argumentations touchant au racisme s'avère adéquate pour discuter les propriétés symboliques de ces argumentations. Au préalable, nous noterons que le caractère transversal de ces processus de catégorisation pointe la dimension typique des cas retenus dans le corpus. Les événements relatés dans les articles du corpus semblent bien relever globalement du phénomène du racisme, et le recours à la classe sociale et sa justification apparaissent comme l'un des registres légitimes pour l'interpréter. Ce constat appuie les remarques de Balibar quant à la tendance universalisante du racisme, qui finit par être présent dans des situations de plus en plus nombreuses (Balibar 2005). Nous avons souligné que ce recours ordinaire à la catégorisation sociale se retrouve de manière importante dans notre corpus, ce qui nous amène à poser que pour l'individu, la définition et la dénonciation ordinaires du racisme via la position 
sociale ont tendance à aller de soi. Toutefois, des positions différentes s'affrontent par leurs modes de raisonnement, les formes et contenus des catégorisations sociales utilisés et les structures de pertinence invoquées, pour, in fine, se disputer la question de la distribution sociale du racisme. Dès lors, la dimension morale propre aux catégories utilisées d'une part et leur caractère exogène de l'autre nous paraissent significatifs pour comprendre l'adéquation entre catégorisations sociales, frontières morales et antiracisme ordinaire.

L'analyse des commentaires à partir des théorisations et des dénonciations ordinaires du racisme nous a amené à prendre en compte le rôle et l'importance des frontières morales construites par les commentateurs. Michèle Lamont, qui a théorisé ce concept de frontières dans la perspective de la sociologie culturelle, s'est également interrogée sur ce lien entre racisme et moralité mais au sein des processus par lesquels les frontières morales entretiennent les barrières entre blancs et noirs, celles-ci étant entendues comme un des socles du racisme lui-même. Dans cet article, nous cherchons plutôt à discuter les façons dont la moralité - c'est-à-dire les frontières morales s'immisce dans les catégorisations opérées par les individus pour définir ceux qui font le racisme. Bien que différentes, ces deux approches nous semblent complémentaires pour embrasser un peu plus la compréhension du phénomène du racisme et de ses implications. Les processus de catégorisations décrits ici sont finalement semblables à ceux décrits par Lamont : discutant un phénomène qui établit des frontières - morales notamment - entre personnes appartenant à des groupes ethniques perçus comme différents, les individus reconstruisent ces même frontières morales pour discuter et dénoncer ce phénomène. La sociologue met en avant le rôle des frontières symboliques au sein du racisme, en tant que processus de justification, par le fait que les « critères utilisés pour évaluer "l'autre" (en termes raciaux) sont souvent une extension de ceux utilisés pour évaluer les "autres" en général » et de là, "les sentiments racistes sont souvent vus comme justifiés, car basés sur des critères universels plutôt que perçus comme expression de préjugés personnels » (Lamont, $2000: 86$ ).

Ces remarques s'appliquent dans une certaine mesure aux registres observées dans les commentaires. Dans l'explication du racisme comme attribut de la classe populaire, nous avons vu par exemple que la théorisation ordinaire passe au préalable par des réflexions concernant la structure sociale et sa hiérarchie d'un côté part, et la conjoncture économique de l'autre. Cependant, c'est dès lors vers des considérations touchant aux frontières morales que s'orientent les argumentations. Usant de critères tels que le manque de culture, l'individualisme et la «fermeture " pour situer la classe populaire, les discours s'appuient finalement sur des critères universels et " objectifs » pour justifier ce rapprochement. La situation économique invoquée sert davantage de prétexte pour spécifier ensuite les traits "naturels » de la classe populaire et leurs superpositions avec ce qui définit le racisme. De manière générale, les frontières morales utilisées pour définir tant le couple classe populaire/élites que le couple beauf/ bobo viennent objectiver et donc justifier les superpositions entre une certaine position sociale et la responsabilité du racisme.

Le second point que soulève cette exploration des processus de catégorisation contenus dans les commentaires est leur caractère exogène. Les constructions analysées ne renvoient ni à des classes en soi ni à des classes pour soi, mais à des processus d'assignation sociale. En mobilisant les théorisations ordinaires et les «figures sociales" observées, les commentateurs catégorisent des comportements ou des 
personnes à partir de leur origine sociale sans jamais s'inclure eux-mêmes dans l'une ou l'autre de ces catégories. Par ailleurs, la distribution sociale du racisme telle qu'elle est perçue par les participants est largement polarisée entre classe populaire et élites, et respectivement entre beaufs et bobos. Pour autant ces catégories s'utilisent toujours par assignation et non par autodéfinition. Ce trait peut a priori s'expliquer par l'absence de face-à-face propre à l'espace des commentaires : dans cet espace, la position sociale est exclusivement une dimension de l'identité sociale virtuelle (Goffman, 1979) et incarne donc principalement une ressource disponible et légitime pour interpréter, catégoriser puis objectiver la position d'autrui. Cependant, les commentateurs, usant de cette position objectivante, ne donnent jamais à voir l'origine sociale de leur propre point de vue. Les commentateurs dénonçant un racisme des élites par un processus de montée en singularité n'incluent pas pour autant leurs propres expériences dans une classe particulière qui partagerait un vécu commun. À l'inverse, leurs propos visent à critiquer les élites, entendues comme une catégorie objective. C'est en cela que la classe sociale, dans son utilisation ordinaire face à la question du racisme, semble avoir pour principale fonction de qualifier et d'évaluer la position d'autrui et ce sans pour autant donner à voir la position de celui qui l'utilise. Pour reprendre la remarque de Lamont concernant l'universalité apparente des critères censés définir l'autre, le caractère exogène des classes tel qu'il apparaît dans les commentaires confère d'autant plus de légitimité aux propos de leurs auteurs qu'ils se placent en dehors de toute appartenance sociale et par extension de tout lien avec le racisme.

C'est donc ce processus d'objectivation, résultat des frontières morales érigées implicitement et du caractère exogène des catégories utilisées, qui fait de la classe sociale un procédé, pour expliquer et dénoncer le racisme, fréquent et d'une certain façon allant de soi. Les processus de catégorisation aperçus dans les commentaires, parfois avec une rare économie de moyens comme pour les figures du beauf et du bobo, permettent donc à la fois d'expliquer, de dénoncer le racisme et même de s'en exonérer, à partir d'une analogie entre les frontières morales de classes et les critères moraux désignant le racisme. Si l'on s'essaie alors à une critique de l'antiracisme ordinaire, on peut d'abord pointer la tendance au «dépistage » propre à ce dernier (Mannheim, 2006 [1929]: 33) ${ }^{11}$. En effet, il y a dans les commentaires étudiés une impossibilité à penser le racisme comme une idéologie totale - via notamment l'idée de racisme structurel (Bonilla-Silva, 1997) - et, à l'inverse, une tendance générale à renvoyer le phénomène à des propriétés individuelles - via des frontières morales notamment ${ }^{12}$. Le "raciste» reste un individu prisonnier d'une idéologie particulière inhérente à sa position sociale jugée déterminante et "totale ", cette essentialisation de la classe n'étant pas sans rappeler les processus typiques du phénomène du racisme décrits par Memmi et Guillaumin.

En définitive, si le racisme repose sur la fabrication de barrières morales entre groupes définis racialement, l'antiracisme ordinaire s'emploie lui aussi à construire des démarcations morales entre les personnes pour catégoriser celles qui sont racistes. En mobilisant ces processus, les commentaires analysés réitèrent alors les critères qui soutiennent certaines des barrières morales propres au racisme. On y remarque au passage, et bien que son exclusion soit à l'origine de ces jeux d'assignations, que le " racisé » semble rester en dehors des catégorisations et figures sociales utilisées par les commentateurs: une autre tendance paradoxale de l'antiracisme ordinaire - qu'il conviendrait évidemment de vérifier par d'autres études - ne serait-elle donc pas de 
catégoriser, voire d'essentialiser, le racisé uniquement à partir de sa supposée différence culturelle?

\section{BIBLIOGRAPHIE}

ADORNO Théodore (2007). Études sur la personnalité autoritaire, Paris, Allia [1950].

ATIFI Hassan \& MARCOCCIA Michel (2005). « Communication médiatisée par ordinateur et variation culturelle : analyse contrastive de forums de discussion français et marocains ", Les Carnets du Cediscor, 9, pp. 59-73.

BALIBAR Étienne (2005). « La construction du racisme », Actuel Marx, 38 (2), pp. 11-28.

BALIBAR Etienne \& WALLERSTEIN Immanuel (1997). Les identités ambigües, La Découverte, Paris. BERGER Peter \& LUCKMANN Thomas (1986). La construction sociale de la réalité, Paris, Klincksieck, [1966].

BOLTANSKI Luc (2009). De la critique, Paris, Métailié.

BOLTANSKI Luc \& THÉVENOT Laurent (1991). De la justification : les économies de la grandeur, Paris, Gallimard.

BONILLA-SILVA Eduardo (2009). Racism without racists: Color-blind racism and the persistence of racial inequality in the United States, New York/Oxford, Rowman \& Littlefield.

BONILLA-SILVA Eduardo (1997). « Rethinking Racism: Toward a Structural Interpretation », American Sociological Review, 62 (3), pp. 465-480.

DUPRET Baudoin (2010). «Commenter l'actualité sur Internet : la structure d'intelligibilité d'un forum de discussion arabe ", Réseaux, 160-161, pp. 290-317.

EBERHARD Mireille (2010). « De l'expérience du racisme à sa reconnaissance comme discrimination. Stratégies discursives et conflits d'interprétation », Sociologie, 1 (4), pp. 479-495.

ELIASOPH Nina (1999). « "Everyday Racism” in a Culture of Political Avoidance : Civil Society, Speech, and Taboo », Social Problems, 46 (4), pp. 479-502.

FALGUÈRES Sophie (2008). Presse quotidienne nationale et interactivité : trois journaux face à leurs publics, Clermont-Ferrand, Presses universitaires Blaise Pascal.

FASSIN Didier (2002). «L'invention française de la discrimination », Revue Française de Science Politique, 52 (4), pp. 403-423.

FASSIN Eric (2006). "Aveugles à la race ou au racisme ? Une approche stratégique », in Fassin Didier et Fassin Eric (dir.), De la question sociale à la question raciale, Paris, La Découverte, pp. 106-130.

GOFFMAN Erving (1979). La mise en scène de la vie quotidienne : Relations en public, Paris, Minuit. GOFFMAN Erving (1984). Les Rites d'interaction, Minuit, Paris. 
GUENIF-SOULAMAS Nacira (2003). « L'irrésistible ascension d'une figure sociale, variations sur le thème du "beauf" ", Ville, école, intégration, 133, pp. 61-73.

GUILLAUMIN Colette (1972). L'Idéologie raciste, Genèse et langage actuel, Paris/La Haye, Mouton. HOGGART Richard (1991). La culture du pauvre, Paris, Minuit.

LAMONT Michèle (2000). La dignité des travailleurs, Paris, Presse de Sciences Po.

LAMONT Michèle (1995). La Morale et l'Argent. La culture des cadres en France et aux États-Unis, Paris, Métailié.

LÉVI-STRAUSS Claude (1952). Race et histoire, Paris, Unesco.

LÉVI-STRAUSS Claude (1971). « Race et culture », Revue internationale des sciences sociales, 23 (4), pp. 647-666.

MANNHEIM Karl (2006 [1929]). Idéologie et utopie, Paris, Éditions de la Maison des Sciences de l'Homme.

MEMMI Albert (1994). Le racisme, Paris, Gallimard.

scHÜTZ Alfred (2008). Le chercheur et le quotidien : phénoménologie des sciences sociales, Paris, Klincksieck.

scHÜTZ Alfred (2007). Essais sur le monde ordinaire, Paris, Le Félin.

TAGUIEFF Pierre-André (1990). La force du préjugé, Paris, Gallimard.

TAGUIEFF Pierre-André (1995). Les fins de l'antiracisme, Paris, Michalon.

TANDÉ Alexandre (2008). « La notion de discrimination dans les discours de l'action publique en France (1992-2005) », Informations Sociales, 148, pp. 20-31.

VAN DIJK Teun A. (1992). « Discourse and the denial of racism », Discourse \& Society, 3 (1), pp. 87-118.

WIEVIORKA Michel (1998). Le racisme, une introduction, La Découverte, Paris.

\section{ANNEXES}

\section{Liste des événements/articles retenus}

\section{Violence racistes}

\section{Meurtre d'un vigile à Bobigny}

http://www.liberation.fr/societe/0101628305-said-noye-par-la-violence-et-la-haine http://www.lefigaro.fr/actualite-france/2010/04/02/01016-20100402ARTFIG00726mort-d-un-vigile-a-bobigny-les-circonstances-se-precisent-.php

\section{Le procès de Youssouf Fofana et le " gang des barbares »}

http://www.liberation.fr/societe/0101564688-a-l-ouverture-de-son-proces-fofanadefie-les-juges 
http://www.lefigaro.fr/actualite-france/2009/04/29/01016-20090429ARTFIG00610fofana-provoque-les-juges-de-la-cour-d-assises-.php

\section{Propos racistes et/ou discriminants}

\section{Le " procès Zemmour "}

http://www.liberation.fr/societe/01012320842-eric-zemmour-condamne-pourprovocation-a-la-haine-raciale

http://www.lefigaro.fr/flash-actu/2011/02/18/97001-20110218FILWWW00474discrimination-raciale-zemmour-condamne.php

\section{Propos racistes du Préfet Langlade}

http://www.lefigaro.fr/actualite-france/2009/08/14/01016-20090814ARTFIG00290-unprefet-sanctionne-pour-injures-racistes-.php

http://www.liberation.fr/societe/0101585502-injures-racistes-le-prefet-suspendu-serebiffe

\section{Propos racistes du maire de Gussainville}

http://www.liberation.fr/brut-de-net/06011471-le-derapage-d-un-maire-ump-lors-dun-debat-sur-l-identite-nationale [cet article n'est plus disponible à l'heure de la mise en ligne de ce texte]

http://www.lefigaro.fr/flash-actu/2009/12/02/01011-20091202FILWWW00447-identitenationale-un-maire-ump-derape.php

\section{Débats nationaux}

La « question Rom »

http://www.liberation.fr/societe/0101648317-sarkozy-accuse-de-stigmatiser-les-romset-les-gens-du-voyage

http://www.lefigaro.fr/politique/2010/07/28/01002-20100728ARTFIG00570-romssarkozy-ne-plie-pas-face-a-la-gauche.php

\section{Interdiction de la Burqa}

http://www.liberation.fr/societe/01012294920-le-conseil-constitutionnel-valide-linterdiction-du-voile-integral

http://www.lefigaro.fr/politique/2010/04/21/01002-20100421ARTFIG00252-le-port-dela-burqa-sera-totalement-interdit-.php 


\section{Affaires internationales}

\section{Déclarations d'Angela Merkel sur le multiculturalisme}

http://www.lefigaro.fr/international/2010/10/17/01003-20101017ARTFIG00129-

angela-merkel-admet-l-echec-du-multiculturalisme-allemand.php

http://www.liberation.fr/monde/01012296716-pour-angela-merkel-le-modelemulticulturel-allemand-a-totalement-echoue

\section{Déclaration de David Cameron sur le multiculturalisme}

http://www.liberation.fr/monde/01012318208-david-cameron-reconnait-l-echec-dumulticulturalisme-en-grande-bretagne

http://www.lefigaro.fr/international/2011/02/05/01003-20110205ARTFIG00536cameron-denonce-l-echec-du-multiculturalisme.php

\section{Extrême droite}

\section{Condamnation des affiches de Jean-Marie Le Pen}

http://www.liberation.fr/politiques/01012323032-affiches-non-a-l-islamisme-peine-deprison-requise-contre-le-pen

http://www.lefigaro.fr/politique/2010/02/25/01002-20100225ARTFIG00665-le-fnprovoque-en-copiant-une-affiche-suisse-antiminarets-.php

\section{Salut nazi d'un candidat du Front National}

http://www.lefigaro.fr/politique/2011/03/25/01002-20110325ARTFIG00585-uncandidat-fn-fait-le-salut-nazi-sur-des-photos.php

http://www.liberation.fr/politiques/01012327813-un-candidat-fn-a-grenoblephotographie-faisant-le-salut-nazi

\section{NOTES}

1. Dans cette direction, voir notamment Fassin (2002) ou encore Tandé (2008).

2. Rétrospectivement, l'ouvrage d'Etienne Balibar et Immanuel Wallerstein (1997) a cependant été lui aussi central dans la compréhension de ce type de racisme.

3. Pour une critique de ce choix de Taguieff, voir Fassin (2006).

4. Voir notamment Sophie Falguères (2008).

5. Derrière le site du Monde, en partie payant, qui, depuis 2004, occupe régulièrement la première place du classement selon les rapports mensuels Médiamétrie (http://www.mediametrie.fr) et OJD (observatoire de la diffusion et du contrôle de journaux, de périodiques et de supports de publicité : http://www.ojd-internet.com ).

6. Pour une présentation de l'interface d'un espace de commentaire et de ses implications voir Dupret (2010).

7. Sur la variation culturelle des échanges informatisés voir Atifi et Marcoccia (2006). 
8. Informations obtenues à partir d'un entretien avec le responsable d'une entreprise de modération importante.

9. Nous n'avons pas pu obtenir cette charte, nettement plus exhaustive que celle proposé aux internautes, auprès de l'entreprise évoquée dans la note précédente et cela en partie pour des questions marketing et concurrentielles.

10. Les commentaires illustrant nos propos ont été anonymisés lorsqu'un pseudonyme apparaissait dans le corps du message ; l'orthographe et la ponctuation ont été conservées.

11. "Cette tendance au dépistage (debunking) est devenue très marquée dans la pensée de notre temps. Et même, dans de nombreux cercles, ce trait est considéré comme manquant de dignité et de respect (et, de fait, si le "dépistage" devient une fin en soi, la critique est justifiée), cette position intellectuelle nous est imposée dans une époque de transition comme la nôtre qui trouve nécessaire de rompre avec bien des traditions et formes vétustes » (Mannheim, 2006 : 33).

12. Nous nous rapprochons ici de la conclusion de Mireille Eberhard (2010) qui, étudiant le racisme et les réponses apportées dans une arène plus "officielle ", à savoir dans le cadre de dispositifs de recueil et de traitement de plaintes individuelles, note que " le moralisme qui imprègne une conception dominante de l'antiracisme et de la lutte contre la discrimination s'accompagne, la plupart du temps, d'une individualisation de la culpabilité de manière à pouvoir personnifier, et donc circonscrire le mal ».

\section{RÉSUMÉS}

Qu'ils incorporent ou non l'idée de classe sociale, quelle(s) représentation(s) les individus se fontils de la structure de la société dans laquelle ils vivent? À quelles occasions et comment mobilisent-ils leurs conceptions de la stratification sociale? Les discussions autour du racisme qui ont lieu en ligne en sont un exemple, comme le montre cet article qui a pour point de départ l'analyse de l'«antiracisme ordinaire" contemporain:l'étude d'un corpus de commentaires d'internautes sur des sites de presse français, dans une perspective inspirée par la sociologie phénoménologique d'Alfred Schütz et le courant pragmatique, révèle en effet que les individus recourent fréquemment à des catégorisations sociales pour dénoncer ce qu'ils perçoivent comme du racisme dans le discours d'autrui. Après avoir rappelé les différentes théories du racisme et de l'antiracisme et discuté de leur pertinence dans l'exploration de cet espace textuel et dialogique particulier, l'article pointe deux types d'argumentation où la position sociale apparaît centrale : l'une fondée sur les connaissances et les capacités réflexives supposées des classes populaires ou des élites, l'autre sur le recours à des figures telles que le « beauf » ou le « bobo ».

Which representation(s) do individuals have of the structure of the society they live in, using or not the idea of social class? How and when do they mobilize their own conceptions of social stratification? Online discussions about racism seem interesting to observe such ordinary knowledge in action. This article deals with the issue of contemporary 'ordinary anti-racism' through the study of a collection of online comments published on French press websites. Inspired by Alfred Schütz's phenomenological sociology and the pragmatic approach, the study reveals that, in fact, individuals often use social categorizations, through various forms, to define and denounce what they perceive as racism. After a review of the different theories of racism and anti-racism, and a discussion about their relevance on this specific, textual and interactional, space, the article explores two types of ordinary repertoire where references to social 
stratification and its boundaries look central to discuss and oppose racism. While the first is focusing on the supposed knowledge and reflexive capacities of the working class and/or the elite, the second is built through the uses of officious social categories of 'beauf'(roughly translated as 'redneck') and 'bobo'

INDEX

Mots-clés : antiracisme, connaissance ordinaire, Internet, racisme, stratification sociale Keywords : antiracism, ordinary knowledge, Internet, racism, social stratification

\section{AUTEUR}

\section{MATTHIEU MAZZEGA}

Université de Grenoble/Institut d'études politiques de Grenoble, PACTE 